\title{
EXPONENTIAL HERMITE SPLINES FOR THE ANALYSIS OF BIOMEDICAL IMAGES
}

\author{
Virginie Uhlmann*, Ricard Delgado-Gonzalo*, Costanza Conti ${ }^{\dagger}$, Lucia Romani ${ }^{\ddagger}$, and Michael Unser* \\ *Biomedical Imaging Group, École polytechnique fédérale de Lausanne (EPFL), Switzerland \\ ${ }^{\dagger}$ Dipartimento di Ingegneria Industriale, Università di Firenze, Italy \\ ${ }^{\ddagger}$ Department of Mathematics and its Applications, University of Milano-Bicocca, Italy
}

\begin{abstract}
We present a new exponential B-spline basis that enables the construction of active contours for the analysis of biomedical images. Our functions generalize the well-known polynomial Hermite B-splines and provide us with a direct control over the tangents of the parameterized contour, which is absent in traditional spline-based active contours. Our basis functions have been designed to perfectly reproduce elliptical and circular shapes. Moreover, they can approximate any closed curve up to arbitrary precision by increasing the number of anchor points. They are therefore well-suited to the segmentation of the roundish objects that are commonly encountered in the analysis of bioimages. We illustrate the performance of an active contour built using our functions on some examples of real biological data.
\end{abstract}

Index Terms - Exponential Hermite splines, parameterization, active contour, segmentation, parametric snake.

\section{INTRODUCTION}

Automated segmentation of biomedical images is a major challenge that is often addressed using active contours. In particular, parametric active contours, referred to as "snakes" [1], are efficient tools for such tasks. A large variety of such models have been presented in the literature [2, 3, 4]. While the design of active contours differs in each case, a two-step algorithmic structure is conserved. In a first step, the snake is initialized by the user or another detection scheme. Then, the curve is made to evolve on the image through the optimization of a cost functional [5].

Our contribution is a class of active contours built using a novel kind of exponential B-spline basis functions. The snakes we introduce have two main features that make them well-suited to the analysis of bioimages. Firstly, we rely on two complementary basis functions, a construction inspired from Hermite interpolation [6] that grants direct control both on the curve and on its tangent field. It therefore enables a

This work was funded by the Swiss SystemsX.ch initiative under Grant 2008/005 and the Swiss National Science Foundation under Grants 200020144355 and 200020-121763.

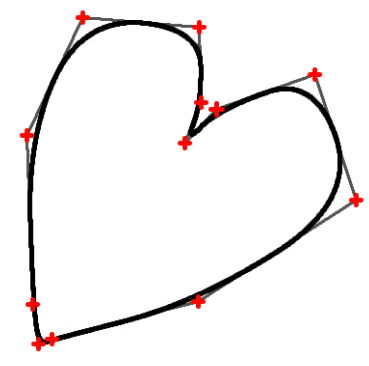

Traditional Spline-Based Snake

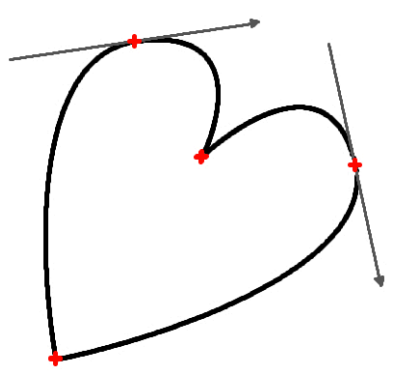

Exponential Hermite Snake
Fig. 1. Comparison of the ability of the proposed parametric snake to approximate an arbitrary closed curve against traditional spline-based parametric snakes. Due to its additional tangential control, our model is able to generate complex shapes using fewer parameters than the traditional splinebased snakes specified by their control polygon.

Bézier-type representation where complex shapes can be generated relying on fewer anchor points than traditional splinebased parametric snakes, as pictured in Figure 1. Secondly, it has ellipse-reproducing capabilities. This property is very desirable as elliptical and round elements such as cells, nuclei, or cross-sections of tubes are common in biomedical images. The method we propose is therefore attuned to a wide range of segmentation tasks.

Our snake model allows for extensive user interaction with the contour while keeping the segmentation process mostly automated. Furthermore, because our functions are interpolating by design, the contour can be precisely edited. The approach was implemented as a plugin for the opensource image-analysis software ImageJ [7] and is available online $^{1}$ for use by the bioimage-analysis community.

In this paper, we first define the Hermite exponential Bsplines we propose, and specify some of their key properties. Then, we introduce the active-contour model built using these functions. Finally, we provide evidence of the efficiency of our method by showing segmentation results on real biological data.

\footnotetext{
${ }^{1}$ http://bigwww.epfl.ch/algorithms/hermitesnake/
} 

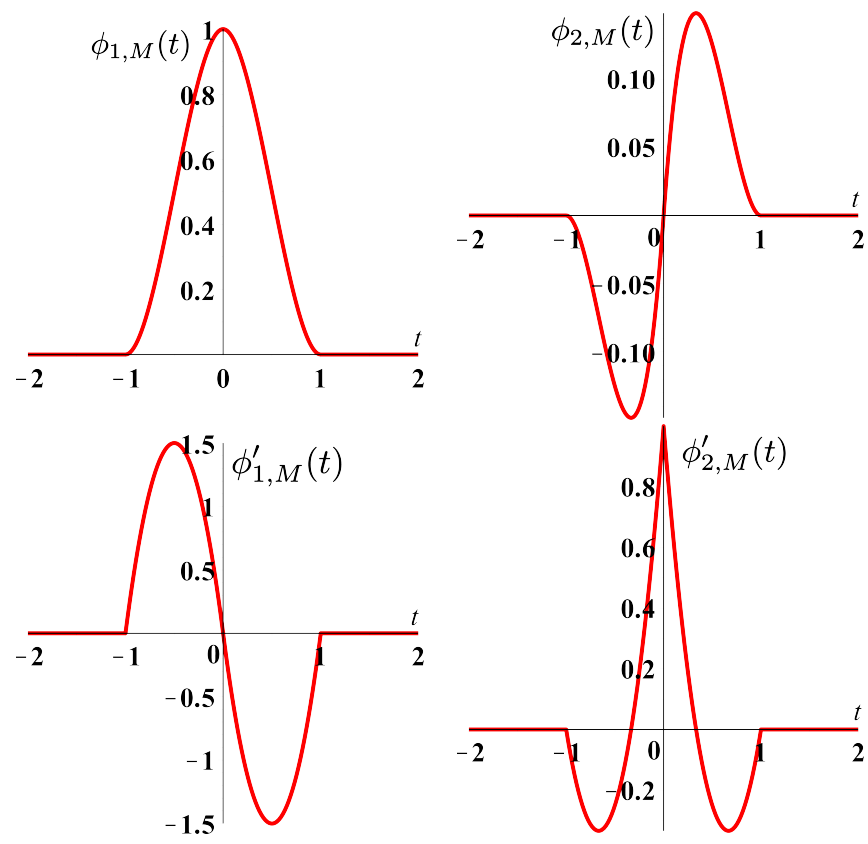

Fig. 2. Ellipse-reproducing Hermite exponential B-spline basis functions (represented for $M=20$ ).

\section{HERMITE EXPONENTIAL B-SPLINES}

We have designed new exponential Hermite spline functions, which generalize the classical Hermite polynomial splines introduced by Lipow and Schoenberg [8]. They are specified by the two basis functions

$$
\begin{aligned}
& \phi_{1, M}(t)= \begin{cases}g_{1, M}(t) & t \geq 0 \\
g_{1, M}(-t) & t<0\end{cases} \\
& \phi_{2, M}(t)= \begin{cases}g_{2, M}(t) & t \geq 0 \\
-g_{2, M}(-t) & t<0\end{cases}
\end{aligned}
$$

built using the generator functions

$$
\begin{aligned}
& g_{1, M}(t)= \begin{cases}a_{1}(M)+b_{1}(M) t+c_{1}(M) \mathrm{e}^{\mathrm{j} \frac{2 \pi}{M} t}+d_{1}(M) \mathrm{e}^{-\mathrm{j} \frac{2 \pi}{M} t} & 0 \leq t \leq 1, \\
0 & \text { elsewhere }\end{cases} \\
& g_{2, M}(t)= \begin{cases}a_{2}(M)+b_{2}(M) t+c_{2}(M) \mathrm{e}^{\mathrm{j} \frac{2 \pi}{M} t}+d_{2}(M) \mathrm{e}^{-\mathrm{j} \frac{2 \pi}{M} t} & 0 \leq t \leq 1, \\
0 & \text { elsewhere }\end{cases}
\end{aligned}
$$

where $M$ is a positive integer. The coefficients of the generators can be expressed as

$$
\begin{array}{rlrl}
a_{1}(M) & =\frac{\mathrm{j} \frac{2 \pi}{M}+1+\mathrm{e}^{\mathrm{j}} \frac{2 \pi}{M}\left(\mathrm{j} \frac{2 \pi}{M}-1\right)}{q(M)} & b_{1}(M) & =-\frac{\mathrm{j} \frac{2 \pi}{M}\left(\mathrm{e}^{\mathrm{j}} \frac{2 \pi}{M}+1\right)}{q(M)} \\
c_{1}(M) & =\frac{1}{q(M)} & d_{1}(M) & =-\frac{\mathrm{e}^{\mathrm{j} \frac{2 \pi}{M}}}{q(M)} \\
a_{2}(M) & =\frac{p(M)}{\mathrm{j} \frac{2 \pi}{M}\left(\mathrm{e}^{\mathrm{j}} \frac{2 \pi}{M}-1\right) q(M)} & b_{2}(M)=-\frac{\mathrm{e}^{\mathrm{j} \frac{2 \pi}{M}}-1}{q(M)} \\
c_{2}(M)=\frac{\mathrm{e}^{\mathrm{j} \frac{2 \pi}{M}}-\mathrm{j} \frac{2 \pi}{M}-1}{\mathrm{j} \frac{2 \pi}{M}\left(\mathrm{e}^{\mathrm{j} \frac{2 \pi}{M}}-1\right) q(M)} & d_{2}(M)=-\frac{\mathrm{e}^{\mathrm{j} \frac{2 \pi}{M}}\left(\mathrm{e}^{\mathrm{j} \frac{2 \pi}{M}}\left(\mathrm{j} \frac{2 \pi}{M}-1\right)+1\right)}{\mathrm{j} \frac{2 \pi}{M}\left(\mathrm{e}^{\mathrm{j}} \frac{2 \pi}{M}-1\right) q(M)},
\end{array}
$$

where

$$
\begin{aligned}
p(M) & =\mathrm{j} \frac{2 \pi}{M}+1+\mathrm{e}^{\mathrm{j} \frac{4 \pi}{M}}\left(\mathrm{j} \frac{2 \pi}{M}-1\right) \\
q(M) & =\mathrm{j} \frac{2 \pi}{M}+2+\mathrm{e}^{\mathrm{j} \frac{2 \pi}{M}}\left(\mathrm{j} \frac{2 \pi}{M}-2\right) .
\end{aligned}
$$

We display $\phi_{1, M}$ and $\phi_{2, M}$ along with their derivatives in Figure 2. The support of these two functions is limited to the interval $[-1,1]$. The fundamental property of this construction is that the generating functions $\phi_{1, M}$ and $\phi_{2, M}$ and their derivatives $\phi_{1, M}^{\prime}$ and $\phi_{2, M}^{\prime}$ satisfy the joint interpolation conditions

$$
\begin{array}{ll}
\phi_{1, M}(n)=\delta_{n} & \phi_{2, M}(n)=0 \\
\phi_{1, M}^{\prime}(n)=0 & \phi_{2, M}^{\prime}(n)=\delta_{n}
\end{array}
$$

for all $n \in \mathbb{Z}$. These basis functions also have the property of reproducing the function space $\left\{1, t, \mathrm{e}^{\mathrm{j} \frac{2 \pi t}{M}}, \mathrm{e}^{-\mathrm{j} \frac{2 \pi t}{M}}\right\}$. Hence, they can be used to generate ellipsoid curves. More mathematical properties of the cardinal Hermite exponential splines, including the associated vector subdivision scheme, are discussed and investigated in the research note [9]. Finally, since

$$
\begin{aligned}
\forall t \in \mathbb{R} \quad: \quad & \sum_{k=-\infty}^{\infty} \phi_{1, M}(t-k)=1 \\
& \sum_{k=-\infty}^{\infty} \phi_{2, M}(t-k)=0,
\end{aligned}
$$

the partition of unity condition is verified [10], which is fundamental for ensuring affine invariance. Any curve parameterized by our exponential Hermite splines will therefore be position- and orientation-independent.

\section{PARAMETRIC HERMITE SNAKE}

Here, we introduce an active contour capable of representing closed curves using the two complementary basis functions (1). The snake is defined entirely by an $M$ periodic sequence of anchor points $\{\mathbf{r}[k]\}_{k \in \mathbb{Z}}$ and tangent vectors $\left\{\mathbf{r}^{\prime}[k]\right\}_{k \in \mathbb{Z}}$, with $\mathbf{r}[k]=\mathbf{r}[k+M]=\left.\mathbf{r}(t)\right|_{t=k}$ and $\mathbf{r}^{\prime}[k]=\mathbf{r}^{\prime}[k+M]=\left.\mathbf{r}^{\prime}(t)\right|_{t=k}$. The contour is then represented by the continuous-domain parameterization

$$
\begin{aligned}
\mathbf{r}(t)= & \sum_{k=0}^{M-1} \mathbf{r}[k] \phi_{1, M, \mathrm{per}}(t-k) \\
& +\mathbf{r}^{\prime}[k] \phi_{2, M, \mathrm{per}}(t-k),
\end{aligned}
$$

where $t \in[0, M]$. Since our construction is designed to produce closed contours, we rely on $M$-periodized versions of our basis functions $\phi_{1, M}$ and $\phi_{2, M}$ that we denote by

$$
\begin{aligned}
\phi_{1, M, \mathrm{per}}(t) & =\sum_{n=-\infty}^{\infty} \phi_{1, M}(t-M n) \\
\phi_{2, M, \mathrm{per}}(t) & =\sum_{n=-\infty}^{\infty} \phi_{2, M}(t-M n) .
\end{aligned}
$$




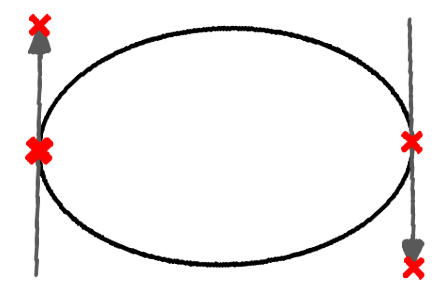

Fig. 3. Reproduction of an ellipse using the proposed exponential Hermite snake.

The positive integer $M$ determines the flexibility of the shape: smaller values of $M$ lead to constrained shapes, while larger ones accommodate more general curves. Therefore, using this model, any closed shape can be approximated to an arbitrary level of accuracy by simply increasing $M$.

This model is reminiscent of the exponential-splines snake (referred to as E-snake) proposed by [11] in the sense that it also allows perfect ellipse reproduction. The novel aspect is the second basis function $\phi_{2, M}$ which brings explicit control over the tangents. The ability to add constraints on the derivative of the curve increases the interaction potential of the snake. This aspect, combined with the interpolation properties of our basis functions, greatly facilitate the design of a flexible user interface, as illustrated in Figure 1. We show in Figure 3 how only two anchor points and their tangents are required to reproduce ellipses. In such case, the generators of the exponential Hermite splines reduce to

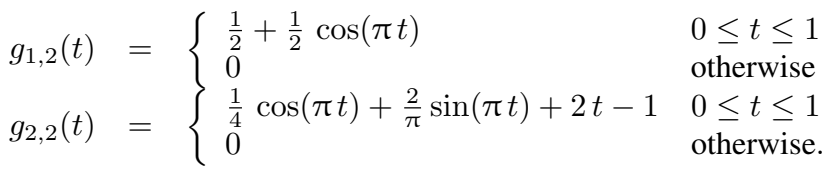

The explicit presence of tangents in our model immediately reminds of the Bézier representation. Yet, the functions are different since they are piecewise exponentials as opposed to piecewise polynomials. In fact, our model uses the shortestpossible functions that have the Hermite property, satisfy the partition of unity, and reproduce ellipses.

\section{EXPERIMENTS}

We demonstrate potential applications of our exponential Hermite snake in microscopic images. We present results on two datasets consisting of images of roundish objects. The images we use are actual biological data. As such, they are representative of the challenges encountered in the real world. In either case, automatic segmentation was performed in a matter of seconds.

\subsection{Segmentation of Phase-Contrast Microscopic Images}

We first assess the efficiency of our snake model in segmenting phase-contrast microscopic images featuring C. Elegans embryo at the 1-cell stage. Data obtained with this imaging modality are challenging for automatic analysis because of the inherent "oriented shading" effect. Indeed, the outline of a cell does not appear as an edge with the same polarity around the whole cell. Most segmentation approaches based on classical edge detectors are unsuccessful on such images. Our Hermite snake is however able to capture the shape of the cell and segment it even with a rough initialization. A sample of the results we obtained is shown in Figure 4. As the C. Elegans embryo is composed of only one elliptical cell at this developmental stage, we initialized our active contour with $M=2$ anchor points in each case.

\subsection{Outline of the Nucleus of a Cell in Fluorescence Im- ages}

Our second dataset consists of fluorescence images featuring nuclei of mouse fibroblast. The objects appear more or less faint depending on the expression of a given protein. The challenge in these images resides in the fact that cells can adopt various shapes and exhibit ill-defined or discontinuous boundaries. We initialized several contours roughly around nuclei in the image. We adapted the number of anchor points $M$ to the complexity of the shape of the nucleus. After optimization, our Hermite snakes were able to outline objects of interest with a variety of intensity and shapes, as displayed in Figure 5.

\section{CONCLUSION}

In this paper, we introduced new exponential B-splines inspired from the cubic Hermite splines. These result in the specification of complementary basis functions that jointly interpolate contour points and their tangents. The conditions that we have imposed ensure affine invariance and the ability to reproduce ellipses and circles.

Using these functions, we implemented a novel parametric segmentation algorithm for the analysis of bioimages. Our approach benefits from several key advantages. First, the ellipse-reproducing property of our model is well-suited to outlining blob-like objects. Then, the additional tangential controls facilitate user-friendly interactions. The applicability of our method was illustrated on real biological data featuring circular or elliptical structures of interest.

\section{REFERENCES}

[1] M. Kass, A. Witkin, and D. Terzopoulos, "Snakes: Active contour models," International Journal of Computer Vision, vol. 1, no. 4, pp. 321-331, January 1988. 

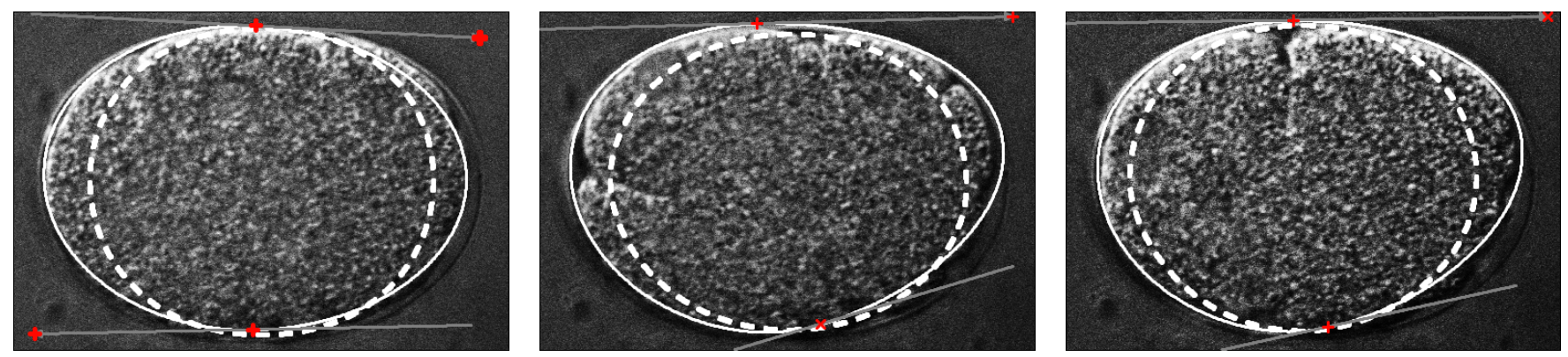

Fig. 4. Segmentation of C. Elegans embryo on phase-contrast microscopic images. Initial elliptical fits are displayed as dashed lines. Results after optimization are shown as solid lines.
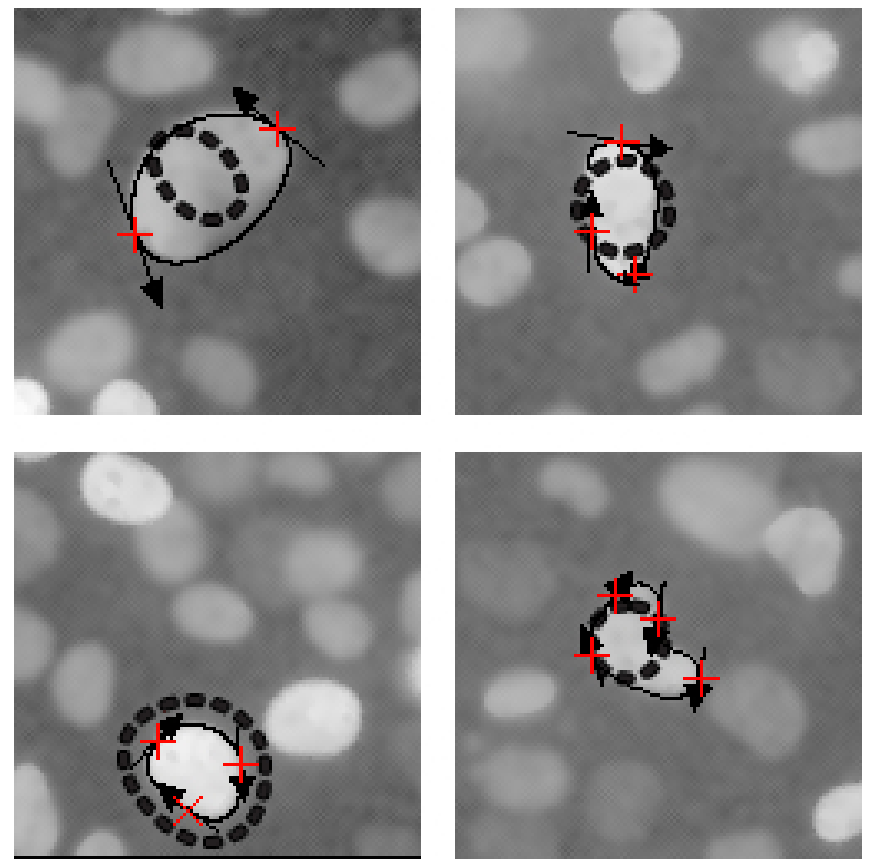

Fig. 5. Outline of mouse fibroblast nuclei in fluorescence microscopic images. Dashed lines correspond to the initial contour of the snake and solid ones to the result obtained after automatic optimization. From left to right, the contour is composed of $M=2, M=3, M=3$, and $M=4$ anchor points.

[2] T. McInerney and D. Terzopoulos, "Deformable models in medical image analysis," in Proceedings of the Workshop on Mathematical Methods in Biomedical Image Analysis (MMBIA'96), San Francisco, CA, 21-22 June 1996, pp. 171-180.

[3] A.K. Jain, Y. Zhong, and M.-P. Dubuisson-Jolly, "Deformable template models: A review," Signal Processing, vol. 71, no. 2, pp. 109-129, December 1998.

[4] S. Menet, P. Saint-Marc, and G. Medioni, "Active contour models: Overview, implementation and applica- tions," in Proceedings of the IEEE International Conference on Systems, Man and Cybernetics (SMC'90). IEEE, 4-7 November 1990, pp. 194-199.

[5] R. Delgado-Gonzalo and M. Unser, "Spline-based framework for interactive segmentation in biomedical imaging," IRBM-Ingénierie et Recherche Biomédicale / BioMedical Engineering and Research, vol. 34, no. 3, pp. 235-243, June 2013.

[6] W. Dahmen, B. Han, R.-Q. Jia, and A. Kunoth, "Biorthogonal multiwavelets on the interval: Cubic Hermite splines," Constructive Approximation, vol. 16, no. 2, pp. 221-259, February 2000.

[7] M.D. Abràmoff, P.J. Magalhães, and S.J. Ram, "Image processing with ImageJ," Biophotonics International, vol. 11, no. 7, pp. 36-42, July 2004.

[8] P.R. Lipow and I.J. Schoenberg, "Cardinal interpolation and spline functions. III. Cardinal Hermite interpolation," Linear Algebra and its Applications, vol. 6, pp. 273-304, 1973.

[9] C. Conti, L. Romani, and M. Unser, "Cardinal Hermite exponential splines," preprint, 2013.

[10] M. Unser, "Sampling-50 years after Shannon," Proceedings of the IEEE, vol. 88, no. 4, pp. 569-587, April 2000.

[11] R. Delgado-Gonzalo, P. Thévenaz, C.S. Seelamantula, and M. Unser, "Snakes with an ellipse-reproducing property," IEEE Transactions on Image Processing, vol. 21, no. 3, pp. 1258-1271, March 2012. 